\title{
The incidence of salmonellosis among dairy herds in the northeastern United States
}

\author{
K. J. Cummings, ${ }^{1}$ L. D. Warnick, K. A. Alexander, C. J. Cripps, Y. T. Gröhn, P. L. McDonough, D. V. Nydam, \\ and K. E. Reed \\ Department of Population Medicine and Diagnostic Sciences, College of Veterinary Medicine, Cornell University, Ithaca, NY 14853
}

\begin{abstract}
The objectives of this study were to estimate the incidence of salmonellosis among a large sample of dairy herds in the northeastern United States (both at the animal level and the herd level), to describe the serotypes and antimicrobial resistance profiles of the positive samples, and to determine whether various herd-level factors were important predictors of incidence. Participating veterinarians enrolled 831 dairy herds and submitted fecal samples from 2,565 female dairy cattle for Salmonella culture because of suspicion of clinical disease. Estimates of animal-level incidence rates were calculated for each age group as the number of cases per animal time at risk, and an estimate of herd-level incidence rate was calculated as the number of positive herds per herd time at risk. Descriptive analysis of serotype data and level of antimicrobial resistance was performed, and Poisson regression analysis was used to study associations between the withinherd incidence of salmonellosis and certain predictor variables (herd size, housing type, vaccination status, and prior history of Salmonella infection). Salmonella was isolated from $576(22.5 \%)$ samples representing 93 herds. The animal-level incidence rates for preweaned female calves, heifers, and adult cows were 8.1, 0.04, and 1.8 cases per 1,000 animal-years, respectively. The herd-level incidence rate was 8.6 positive herds per 100 herd-years. Salmonella Newport was the predominant serotype, accounting for $41 \%$ of the cases, followed by Salmonella Typhimurium. Over $68 \%$ of all isolates were resistant to 5 or more antimicrobial agents. Herd size was the only significant predictor of the incidence of salmonellosis in a multivariable model; herds with at least 400 female dairy cattle had a higher incidence rate than smaller herds. Our results shed light on the impact of salmonellosis on the dairy industry in the
\end{abstract}

Received January 30, 2009.

Accepted March 31, 2009.

${ }^{1}$ Corresponding author: kjc39@cornell.edu northeastern United States, and they help clarify the role of dairy cattle as a source of Salmonella serotypes that are also important human pathogens.

Key words: incidence, salmonellosis, dairy herd

\section{INTRODUCTION}

Salmonella is a zoonotic enteric pathogen that can cause significant disease in both calves and adult cattle. Clinical signs of bovine salmonellosis may include diarrhea, fever, anorexia, dehydration, decreased milk production, abortion, and evidence of endotoxemia, although many infections remain subclinical (Divers and Peek, 2008). It can be a costly disease for dairy producers because of treatment expenses, mortality, reduced milk yield, and weight loss/decreased weight gain within the herd (Peters, 1985; Huston et al., 2002). Salmonella also poses a considerable threat to public health, resulting in approximately 1.4 million illnesses, 16,000 hospitalizations, and between 400 and 600 deaths annually in the United States alone (Mead et al., 1999; Voetsch et al., 2004).

Infected cattle can shed the organism while ill and following clinical recovery, and asymptomatic shedders never show signs. Widespread environmental contamination can result from Salmonella shedding, and the organism can survive for prolonged periods in suitable conditions outside a host (Wray and Wray, 2000). Fecal Salmonella shedding can also augment the risk of within-herd transmission and inadvertent spread to other herds. In addition to having implications for the health and productivity of dairy cattle, these factors lead to an increased risk of zoonotic transmission. Foodborne exposure may occur when there is fecal contamination of beef carcasses at slaughter, contamination of crops by manure fertilizer, and contamination of water by manure run-off (Wells et al., 2001; Islam et al., 2004; Sivapalasingam et al., 2004). People can also become infected via direct contact; those who work or otherwise interact with livestock are at particular risk when cattle are shedding Salmonella. Fecal shedding often persists well beyond the clinical outbreak in the herd (Clegg et 
al., 1983; Giles et al., 1989; Gay and Hunsaker, 1993), underscoring the difficulty in recognizing high-risk herds.

Introduction of Salmonella onto a dairy farm can occur through a variety of routes, including purchased cattle, contaminated feed or water, wild animals such as rodents and birds, and human traffic (Bender, 1994; Evans and Davies, 1996; Sanchez et al., 2002; Nielsen et al., 2007). Therefore, the presence of Salmonella on a farm is not an unexpected finding. In fact, one study involving 110 dairy farms in 4 states found that over $90 \%$ of the farms had at least one Salmonella-positive culture obtained (fecal or environmental) during the course of 5 sampling visits over a 1-yr period (Fossler et al., 2004). The USDA National Animal Health Monitoring System (NAHMS) Dairy 2002 study, based on a single sampling visit to 5 herds in each of 21 states, found that $31 \%$ of herds yielded at least one Salmonellapositive fecal culture (Blau et al., 2005).

Although several studies have examined the prevalence of fecal Salmonella shedding on dairy farms (Wells et al., 2001; Huston et al., 2002; Fossler et al., 2004; Blau et al., 2005), there is very little information available regarding the occurrence of clinical disease associated with Salmonella infections in cattle. Knowledge of the distribution and frequency of salmonellosis, both among herds and among age groups within a herd, would be important for producers and veterinarians alike. An understanding of the full impact of Salmonella on the health of dairy cattle could lead to more focused strategies for preventing the introduction and spread of this pathogen on the farm, with obvious public health benefits as well. Furthermore, information regarding which serotypes are typically associated with clinical disease in cattle would shed light on the risk posed by certain types of Salmonella on the dairy farm; this would also add to our collective knowledge of the role played by dairy cattle as a potential source of Salmonella infection for people. It would be especially helpful to gain information on whether clinically affected cattle are shedding the serotypes that are also important human pathogens, such as Newport and Typhimurium, as this could facilitate the recognition of herds that present the greatest threat to public health. Thus, the objectives of this study were to estimate the animal- and herd-level incidence of salmonellosis among a large sample of dairy herds in the northeastern United States, to describe the serotypes and antimicrobial resistance profiles of the positive samples, and to ascertain whether certain herd-level factors (herd size, housing type, vaccination status, and prior history of Salmonella infection) were important predictors of incidence.

\section{MATERIALS AND METHODS}

\section{Study Design}

Veterinary practices serving New York, Pennsylvania, Vermont, Massachusetts, and Connecticut were enrolled between February and September 2004, with the goal of selecting practices that were known to provide clinical service to a large number of dairy herds. Practices were identified through our personal contacts and via an announcement of the study at a regional veterinary continuing education meeting. Participating veterinarians were asked to enroll all of their dairy herd clients with at least 30 dairy cattle for whom they provided routine clinical service. Veterinarians were instructed to educate their clients regarding the clinical signs of salmonellosis in cattle, including diarrhea with blood, mucus, or a foul odor; fever of at least $103^{\circ} \mathrm{F}$ $\left(39.4^{\circ} \mathrm{C}\right)$; depression; and decreased appetite; as well as sudden death in the absence of specific clinical signs. Herd owners were asked to contact their veterinarian if any of their cattle displayed these signs or if unusually high mortality among cattle with diarrhea was noted. At enrollment, veterinarians collected information on herd size, type of housing, and vaccination protocols. Throughout the duration of the study, project personnel would obtain updated information on numbers of cattle within each herd; the targeted interval between herd updates was 3 mo.

Veterinarians submitted fecal samples from suspected clinical cases to the Animal Health Diagnostic Center at Cornell University for Salmonella culture, from the time of herd enrollment through September 2005. To encourage herd owners and veterinarians to submit samples from every clinical suspect animal, all shipping and laboratory costs were covered by the study. In addition, no costs were incurred by herd owners for the testing of calves and weaned heifers for other enteric pathogens if requested by their veterinarian. Herd owners were also allowed to submit Salmonella fecal culture samples from animals with compatible clinical signs, provided this was done under the supervision of their veterinarian.

\section{Sample Collection and Processing}

Fecal samples were generally collected via rectal retrieval, with a new glove being used to collect each sample. Approximately $10 \mathrm{~g}$ of fecal matter was placed into a Para-Pak bottle (containing modified Cary-Blair medium, Meridian Bioscience Inc., Cincinnati, $\mathrm{OH}$ ) and sealed. For small calves, a BBL culture swab (Amies medium; Becton Dickinson and Company, Franklin 
Lakes, NJ) was occasionally used for sample collection. All samples were either delivered by the participating veterinarian or shipped to the Animal Health Diagnostic Center for bacteriologic culture, and standard culture methods were used to isolate Salmonella from feces. Individual fecal swabs from sample bottles were enriched in $10 \mathrm{~mL}$ of tetrathionate broth (Difco, Detroit, MI) containing $0.2 \mathrm{~mL}$ of iodine solution; the mixture was incubated at $42^{\circ} \mathrm{C}$ for 18 to $24 \mathrm{~h}$. After incubation, the sample-broth mixture was streaked onto brilliant green agar with novobiocin (BGN; Becton Dickinson) and xylose lysine tergitol 4 (XLT-4) selective media, and both plates were incubated at $37^{\circ} \mathrm{C}$ for 18 to $24 \mathrm{~h}$. Red bacterial colonies (lactose-non-fermenting) on BGN and black colonies $\left(\mathrm{H}_{2} \mathrm{~S}\right.$-producing) on XLT-4 were inoculated into Kligler iron agar (KIA) slants and then incubated at $37^{\circ} \mathrm{C}$ for 18 to $24 \mathrm{~h}$. The XLT- 4 plates without suspected colonies were reincubated at $37^{\circ} \mathrm{C}$ for an additional 18 to $24 \mathrm{~h}$ before checking again for characteristic black colonies. Colonies on KIA slants that exhibited the biochemical properties of Salmonella were then serogrouped by slide agglutination using standard protocols. Those colonies that were positive by slide agglutination were then identified as Salmonella using the Sensititre Automated Microbiology System's A80 panel (Trek Diagnostic Systems Inc., Cleveland, OH). Confirmed Salmonella isolates were sent to the USDA National Veterinary Services Laboratories (Ames, IA) for serotyping using standard protocols.

Antimicrobial susceptibility of Salmonella isolates was determined by use of the broth dilution method. Minimal inhibitory concentrations were established for each isolate against a panel of 11 antimicrobial agents (ampicillin, ceftiofur, chlortetracycline, enrofloxacin, florfenicol, gentamicin, neomycin, oxytetracycline, spectinomycin, sulfadimethoxine, and trimethoprim/ sulfamethoxazole; Sensititre, Trek Diagnostic Systems Inc.). Clinical and Laboratory Standards Institute (CLSI) guidelines were used to interpret MIC values when available (CLSI, 2008). Isolates were classified as being resistant or susceptible to each agent; those isolates with intermediate susceptibility were categorized as being susceptible. Quality control was performed weekly using 4 strains of bacteria: Escherichia coli ATCC 25922, Staphylococcus aureus 29213, Enterococcus faecalis 29212, and Pseudomonas aeruginosa 27853. The MIC ranges for quality control recommended by the CLSI were used, and results were accepted if the MIC values were within expected ranges for these bacterial strains.

\section{Data Analysis}

All animals were classified as preweaned female calves, preweaned male calves, heifers (from weaning age - approximately $8 \mathrm{wk}$ - to calving age), adult cows, or adult bulls. Estimates of herd size for incidence calculations were based on the initial total at enrollment and the subsequent updates obtained throughout the study. For each time interval between updates, the number of cattle at risk in each age group was calculated as the average of the number of animals reported at the start and at the end of the interval. In doing this, we accounted for changes in the size of the population at risk due to death, sale, or purchase. Animal time at risk for each age group was calculated by multiplying each herd's number of animals at risk by the time interval between updates, then summing these across all herds; cases were not excluded from the animal time at risk calculations following diagnosis because of their negligible contribution to the total time at risk. Herd time at risk was calculated by summing the total enrollment times for all negative herds and the time between enrollment and onset of the first case for each positive herd. Incident cases were defined as clinically ill cattle that were identified as having signs consistent with salmonellosis and that tested positive for Salmonella via bacteriologic culture. Repeated positive samples from the same animal were excluded from incidence calculations. A herd was considered Salmonella-positive if at least one animal with compatible clinical signs tested positive via bacteriologic culture; such an animal had to have been diagnosed at least 2 wk beyond herd enrollment in order for it to be viewed as an incident case. Estimates of animal-level incidence rates (incidence densities) were calculated for each age group as the number of cases per animal time at risk, and an estimate of herd-level incidence rate was calculated as the number of positive herds per herd time at risk.

To check for selection bias related to herd enrollment, estimates of animal-level incidence rates were also calculated separately for herds within veterinary practices that enrolled more than $50 \%$ of their dairy clients and for herds within practices enrolling fewer than $50 \%$. Our concern was that low-enrollment practices might have been selectively recruiting study herds with either a known history of salmonellosis or a current outbreak of diarrhea, rather than a representative sample of their client herds.

Descriptive analysis of serotype data and level of antimicrobial resistance was performed, including the distribution of serotypes and multidrug-resistant (MDR) isolates by animal age group and by herd. The proportion of MDR isolates by serotype was also determined. Bivariable analysis using the chi-squared test was utilized to determine whether age group or serotype was significantly associated with multidrug resistance (MDR vs. not MDR). Separate logistic regression models were used to further investigate any 
associations with multidrug resistance while controlling for herd as a random effect, using MDR status as the dichotomous outcome variable. In this study, multidrug resistance was defined as having in vitro resistance to 5 or more antimicrobial agents.

Poisson regression analysis was performed to study associations between the within-herd incidence of salmonellosis and various predictor variables (herd size, housing type, vaccination status, and prior history of Salmonella infection), controlling for herd as a random effect. A backward stepwise approach was used to identify a final multivariable model, and $P$-values $<0.05$ were considered significant. Incidence density ratios and their corresponding $95 \%$ confidence intervals were determined. All data analysis was performed in SAS (version 9.1; SAS Institute Inc., Cary, NC), and the generalized estimating equations (GEE) method was used for the regression models via PROC GENMOD.

\section{RESULTS}

A total of 35 veterinary practices participated in this study, enrolling 831 herds with 327,686 female dairy cattle (preweaned female calves, heifers, and adult cows). Median herd size was 180 female dairy cattle (range: 20-5,241). Free-stall housing was found on 454 $(54.6 \%)$ farms (this was the exclusive form of housing on 418 of these farms), while barns with individual stalls for each cow (either tie-stall or stanchion) were utilized on 355 (42.7\%) farms; the remaining $2.7 \%$ of farms used some other form of housing. Use of a commercial gram-negative vaccine was reported among 337 $(40.6 \%)$ herds (Endovac-Bovi, Immvac Inc., Columbia, MO; J-Vac, Merial, Duluth, GA; J-5 Bacterin, Pfizer Animal Health, New York, NY). There were 59 (7.1\%) herds that had reportedly experienced at least one Salmonella case during the previous 12 mo.

During the study period, fecal samples from 2,565 female dairy cattle on 412 farms were submitted for Salmonella culture because of suspicion of clinical disease. Of these, Salmonella was isolated from 576 $(22.5 \%)$ samples representing 93 herds. Sixteen herds accounted for $72 \%$ of the positive samples, whereas 40 herds had only one laboratory-confirmed case of salmonellosis each. Twelve of the 40 herds had their single case diagnosed during the 2 wk immediately following herd enrollment, and those animals were not regarded as incident cases in any of the calculations. The herd-level incidence rate was 8.6 positive herds per 100 herd-years.

Fecal samples were submitted from 866 preweaned female calves, 168 heifers, and 1,531 adult cows for Salmonella culture. The organism was isolated from 152
(17.6\%) female calves, 7 (4.2\%) heifers, and $417(27.2 \%)$ cows. The animal-level incidence rates for preweaned female calves, heifers, and adult cows were 8.1, 0.04, and 1.8 cases per 1,000 animal-years, respectively. For herds within high-enrollment practices, the animal-level incidence rates for preweaned female calves, heifers, and adult cows were 9.9, 0.09 , and 2.0 cases per 1,000 animal-years, whereas herds within low-enrollment practices had incidence rates of $7.4,0.03$, and 1.8 cases per 1,000 animal-years, respectively. The within-herd incidence rates among all positive herds ranged from 0.2 to 119.3 cases per 1,000 animal-years, with a median of 4.8 cases per 1,000 animal-years (Figure 1).

The predominant serotype was Newport, accounting for $41 \%$ of the cases, followed by Typhimurium (including the Copenhagen variant), Infantis, 4,5,12:i:-, Agona, Muenster, and Kentucky. These 7 serotypes comprised $87 \%$ of the total, and 11 other serotypes made up the remainder (Table 1). The serotype most frequently isolated from calves was Typhimurium (40.1\%, 61/152), whereas the primary serotype among cows was Newport $(46.8 \%, 195 / 417)$. The most common serotypes were also widespread among farms, with Typhimurium being isolated from 36 herds and Newport from 30 herds (Table 2). There were $71(76.3 \%)$ herds that had only one serotype identified during the study period, $18(19.4 \%)$ herds had 2 serotypes, and $4(4.3 \%)$ had 3 serotypes.

A total of 395 (68.6\%) isolates were MDR. Only 114 $(19.8 \%)$ isolates were pan-susceptible, not displaying resistance to any of the 11 antimicrobial drugs in our panel. Resistance to individual antimicrobial agents (Table 3 ) ranged from $0 \%$ (enrofloxacin) to $79.3 \%$ of all isolates (sulfadimethoxine). Chi-squared testing revealed that the proportion of multidrug resistance was significantly higher $(P=0.006)$ among isolates obtained from calves $(77.0 \%, 117 / 152)$ than among those from cows $(65.2 \%, 272 / 417)$. Calf isolates were also more likely to be MDR than cow isolates in a logistic regression model that controlled for herd as a random effect $(P=0.01)$. In addition, 6 of the 7 isolates from heifers were MDR. Cattle harboring MDR isolates represented 60 (64.5\%) of the Salmonella-positive herds in our study.

There was considerable variation in antimicrobial resistance across serotypes (Table 4). For instance, 97.0\% (229/236) of the Newport isolates were MDR, whereas only $2.1 \%(1 / 47)$ of the Infantis isolates were MDR. Chi-squared testing showed that the proportion of multidrug resistance was significantly higher $(P<0.0001)$ among serotypes that are also the most important human pathogens (Newport and Typhimurium; 89.0\%, 308/346) than among all other serotypes $(37.4 \%, 86 / 230)$. Newport and Typhimurium isolates 


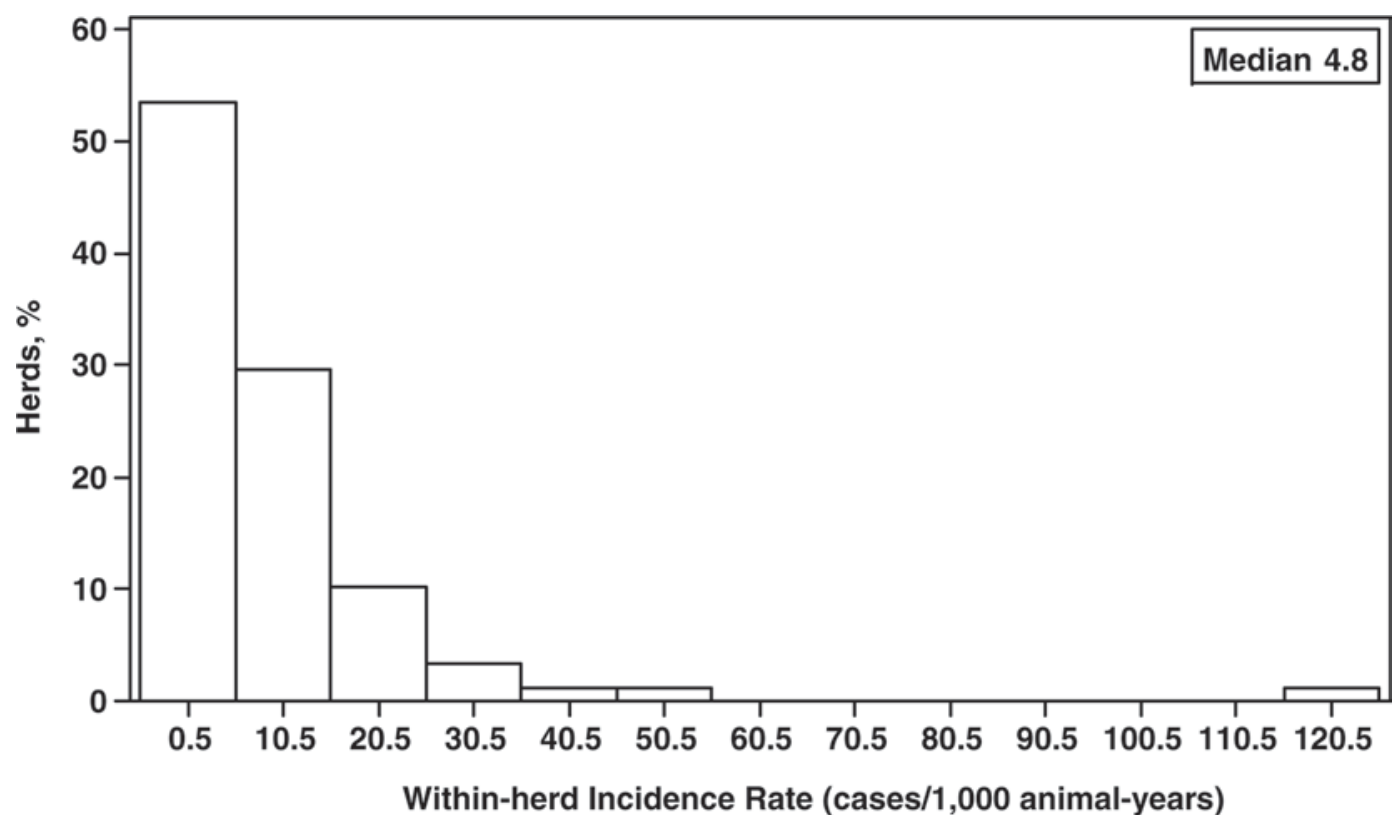

Figure 1. Within-herd incidence rates among all positive herds.

were also more likely to be MDR than other serotypes in a logistic regression model controlling for herd as a random effect $(P<0.0001)$.

Using a multivariable Poisson regression model, we found that herd size was the only significant predictor of Salmonella incidence (Table 5). Larger herds with at least 400 female dairy cattle had a higher incidence rate than smaller herds with fewer than 100 female dairy cattle (incidence density ratio of $4.7 ; P=0.004$ ). The incidence rates among the 3 smaller herd size categories (200-399, 100-199, and <100 female dairy cattle) did not differ significantly. There was not a significant association between Salmonella incidence and housing type, commercial gram-negative vaccine use, or a history of at least one Salmonella case during the previous 12 mo when these were included with herd size in a multivariable model.

\section{DISCUSSION}

Several studies have described the prevalence of fecal Salmonella shedding among dairy cattle, using either cross-sectional or longitudinal designs. To our knowledge, however, no studies have investigated the incidence of clinical disease associated with Salmonella infections in cattle. This study had the particular advantage of involving a very large number of female cattle (over 327,000) from numerous dairy herds (831) throughout the northeastern United States. These herds were characterized by a wide range of sizes and management types representative of the dairy industry in this region of the country. Although our goal was for participating veterinarians to enroll all of their dairy herd clients with at least 30 dairy cattle for whom they provided routine clinical service, not all such herds were enrolled. However, our comparable animal-level incidence rates for herds within high-enrollment and low-enrollment practices would suggest that selection bias related to herd enrollment was not present in this study. In fact, the incidence rates for all animal age groups were actu-

Table 1. Serotypes isolated from female dairy cattle with salmonellosis in the northeastern United States

\begin{tabular}{lr}
\hline Serotype & Isolates, $\mathrm{n}(\%)$ \\
\hline $4,5,12: \mathrm{i}:-$ & $35(6.1)$ \\
Adelaide & $1(0.2)$ \\
Agona & $30(5.2)$ \\
Anatum & $9(1.6)$ \\
Bardo & $5(0.9)$ \\
Cerro & $1(0.2)$ \\
Infantis & $47(8.2)$ \\
Kentucky & $20(3.5)$ \\
Mbandaka & $12(2.1)$ \\
Montevideo & $5(0.9)$ \\
Muenchen & $4(0.7)$ \\
Muenster & $23(4.0)$ \\
Newport & $236(41.0)$ \\
Ohio & $11(1.9)$ \\
Oranienburg & $3(0.5)$ \\
Senftenberg & $1(0.2)$ \\
Thompson & $2(0.3)$ \\
Typhimurium & $49(8.5)$ \\
Typhimurium (Copenhagen) & $61(10.6)$ \\
Unknown & $21(3.6)$ \\
\hline
\end{tabular}


Table 2. Distribution of the most common Salmonella serotypes by dairy herd

\begin{tabular}{lcc}
\hline Serotype & Dairy herds, $\mathrm{n}$ & Positive herds, \% \\
\hline $4,5,12: \mathrm{i}:-$ & 4 & 4.3 \\
Agona & 8 & 8.6 \\
Infantis & 7 & 7.5 \\
Kentucky & 2 & 2.2 \\
Muenster & 6 & 6.5 \\
Newport & 30 & 32.3 \\
Typhimurium & 27 & 29.0 \\
Typhimurium (Copenhagen) & 11 & 11.8 \\
\hline
\end{tabular}

ally lower among practices that were apparently more selective in enrolling herds.

It is conceivable that the incidence of salmonellosis among dairy herds was underestimated if clinically affected cattle went undetected by herd owners. Furthermore, fecal culture does not have perfect sensitivity for detecting the presence of Salmonella, and we recognize that some positive cattle were presumably missed by culturing. On the other hand, it is also plausible that some animals with a positive Salmonella culture result and compatible clinical signs were actually symptomatic because of another primary disease process; this would lead to an overestimation of the incidence of salmonellosis. Alternatively, it is possible that some cases assumed to be incident were in fact ongoing at the start of the study, also leading to an overestimation of incidence rates. However, we believe that we eliminated this possibility by requiring that only cases diagnosed at least $2 \mathrm{wk}$ beyond herd enrollment be considered for incidence calculations.

Laboratory-confirmed salmonellosis was found in $11 \%(93 / 831)$ of the dairy herds monitored for approximately 1 yr over the course of the study. The herd-level incidence rate was approximately 9 positive herds per 100 herd-years. However, only $17 \%$ (16/93) of the positive study herds accounted for over $70 \%$ of the clinical
Salmonella cases. The median within-herd incidence rate among positive herds was approximately 5 cases per 1,000 animal-years, with a maximum of almost 120 cases per 1,000 animal-years. These results suggest a wide disparity in the incidence of salmonellosis among dairy herds in the northeastern United States. Although most farms did not experience any clinical disease due to Salmonella infection during the study period, a subset of the positive farms had a very high incidence of salmonellosis. Clustering of disease among herds is consistent with the prevalence study that found that $25 \%$ of the enrolled dairy farms accounted for over $75 \%$ of the Salmonella-positive fecal and environmental samples (Fossler et al., 2004). It seems likely that the most efficient approach to controlling Salmonella at the farm level would be to focus our efforts on addressing biosecurity and hygiene practices among the relatively few herds with a high frequency of disease, as well as preventing pathogen spread from such herds to those that remain uninfected.

Over $22 \%(576 / 2,565)$ of the fecal samples from clinical suspect animals were positive for Salmonella. The animal-level incidence rate for preweaned female calves was 8.1 cases per 1,000 animal-years, whereas that for adult cows was 1.8 cases per 1,000 animal-years. Heifers in this study rarely developed salmonellosis. Both humoral and cellular immune mechanisms play a role in resistance to Salmonella (Lindberg and Robertsson, 1983), and calves may be at a greater risk of infection than adults because of their more naïve immune systems. In addition, concurrent infection with multiple enteric pathogens (Escherichia coli, Rotavirus, Coronavirus, or Cryptosporidium) is a common scenario in calves (Divers and Peek, 2008), further compromising their immune status. It is also possible that a relative lack of intestinal microflora in young calves contributes to their susceptibility; such microflora is believed to offer a degree of protection against colonization by

Table 3. Resistance to individual antimicrobial agents among all Salmonella isolates and the most common serotypes

\begin{tabular}{|c|c|c|c|c|c|c|c|c|c|}
\hline \multirow[b]{2}{*}{ Antimicrobial agent } & \multicolumn{9}{|c|}{ Resistance, \% } \\
\hline & All isolates & 4,5,12:i:- & Agona & Infantis & Kentucky & Muenster & Newport & Typhimurium & $\begin{array}{l}\text { Typhimurium } \\
\text { (Copenhagen) }\end{array}$ \\
\hline Ceftiofur & 60.4 & 57.1 & 80.0 & 2.1 & 5.0 & 8.7 & 97.0 & 10.2 & 42.6 \\
\hline Chlortetracycline & 66.8 & 62.9 & 80.0 & 4.3 & 0 & 8.7 & 96.6 & 30.6 & 91.8 \\
\hline Enrofloxacin & 0 & 0 & 0 & 0 & 0 & 0 & 0 & 0 & 0 \\
\hline Neomycin & 42.2 & 5.7 & 73.3 & 2.1 & 5.0 & 0 & 55.5 & 22.4 & 78.7 \\
\hline Oxytetracycline & 68.9 & 62.9 & 83.3 & 4.3 & 5.0 & 8.7 & 97.5 & 38.8 & 96.7 \\
\hline Spectinomycin & 16.1 & 8.6 & 10.0 & 2.1 & 0 & 8.7 & 2.1 & 36.7 & 85.2 \\
\hline Sulfadimethoxine & 79.3 & 68.6 & 100.0 & 6.4 & 65.0 & 60.9 & 97.0 & 65.3 & 100.0 \\
\hline Trimethoprim/sulfamethoxazole & 5.2 & 0 & 63.3 & 2.1 & 0 & 4.3 & 1.3 & 2.0 & 9.8 \\
\hline
\end{tabular}


Table 4. Multidrug resistance (MDR; in vitro resistance to $\geq 5$ antimicrobial agents) among the most common Salmonella serotypes

\begin{tabular}{lc}
\hline Serotype & MDR, \% (n/n) \\
\hline $4,5,12: 1:-$ & $60.0(21 / 35)$ \\
Agona & $83.3(25 / 30)$ \\
Infantis & $2.1(1 / 47)$ \\
Kentucky & $5.0(1 / 20)$ \\
Muenster & $8.7(2 / 23)$ \\
Newport & $97.0(229 / 236)$ \\
Typhimurium & $38.8(19 / 49)$ \\
Typhimurium (Copenhagen) & $98.4(60 / 61)$ \\
\hline
\end{tabular}

pathogenic enteric bacteria. Very few studies compare Salmonella prevalence among age groups of cattle, with mixed results regarding whether preweaned calves (Warnick et al., 2003) or cows (Fossler et al., 2005b) have a higher prevalence of fecal Salmonella shedding. Calf isolates in this study were also more likely to be MDR than isolates from cows, a trend that has been observed with E. coli as well (Sato et al., 2005; Cho et al., 2007).

Newport was certainly the major serotype in this study, accounting for over $40 \%$ of the isolates, followed by Typhimurium at nearly $20 \%$; both were widespread among farms. Dairy cattle represent an important source of Salmonella serotypes that are a threat to human health. The Centers for Disease Control and Prevention (CDC) FoodNet data from 2007 show that Newport and Typhimurium were 2 of the 3 most common Salmonella serotypes isolated from people with laboratory-confirmed foodborne infection, accounting for over $26 \%$ of the human cases (CDC, 2008b). The predominance of Salmonella Newport in this study is particularly noteworthy because it is generally MDR in cattle and is becoming an increasingly important human pathogen. According to the CDC, the annual incidence of Salmonella Newport infections among people in the United States has increased by more than $40 \%$ over the last decade (CDC, 2007). Multidrug resistance is also on the rise; the prevalence of the most common MDR Salmonella Newport phenotype (Newport-MDRAmpC, resistant to at least 9 antimicrobial agents) increased from $1 \%$ of human Newport isolates tested by the National Antimicrobial Resistance Monitoring System in 1998 to $21 \%$ of isolates tested in 2003. Reported risk factors for Newport-MDRAmpC infection in people include direct exposure to a dairy farm (Gupta et al., 2003), consumption of uncooked ground beef (Varma et al., 2006), and consumption of unpasteurized dairy products (CDC, 2008a); these examples illustrate the key role that dairy cattle play as a source of MDR Salmonella Newport.

In contrast, the serotypes most commonly isolated in studies of fecal Salmonella shedding among clinically healthy cattle differ from those that most frequently cause human disease. According to the 1996 NAHMS report, Salmonella Montevideo (21\%) was the most prevalent serotype isolated from healthy lactating cows, and neither Newport nor Typhimurium was among the 10 most common serotypes isolated (Wells et al., 2001). The 2002 NAHMS study found Salmonella Meleagridis (24\%) to be the most prevalent serotype, whereas Newport and Typhimurium accounted for only 3 and $10 \%$ of all isolates, respectively (Blau et al., 2005). Clearly, there is a great diversity in Salmonella serotypes shed by dairy cattle. Although many serotypes may be shed by apparently healthy cattle, our results suggest that Newport and Typhimurium are two that pose a higher risk to the health and welfare of cattle. Furthermore, this study supports the view that clinically affected cattle present the greatest threat to public health, as they are often shedding serotypes that are also important human pathogens. This would be in contrast with other foodborne zoonotic pathogens, such as Campylobacter jejuni and E. coli O157:H7, which occur widely in adult cattle without accompanying clinical disease (Cho et al., 2006; Kwan et al., 2008).

Interestingly, the Dublin serotype was not isolated from any of the cattle in this study, which may simply reflect the fact that this serotype was not common among northeastern dairy farms during the study period. Alternatively, this may be related to our emphasis on enteric disease in the case definition provided to herd owners. The predominant clinical sign among cattle infected with Dublin is respiratory disease rather than diarrhea (Divers and Peek, 2008), and we may have missed such cases as a result. Furthermore, optimal culture specimens for diagnosing Salmonella Dublin infections are a trans-tracheal aspirate or blood culture rather than a fecal sample.

Herd size was a significant predictor of the incidence of salmonellosis in a multivariable Poisson regression model. The association between large herd size and fecal Salmonella shedding has been reported in numerous studies (Kabagambe et al., 2000; Warnick et al., 2001; Wells et al., 2001; Huston et al., 2002; Fossler et al., 2004; Blau et al., 2005; Fossler et al., 2005b; Davison et al., 2006). Larger herds may have a greater likelihood of

Table 5. Multivariable Poisson regression analysis of herd-level factors for association with Salmonella incidence rate (incidence density)

\begin{tabular}{lccc}
\hline $\begin{array}{l}\text { Herd size (female } \\
\text { dairy cattle) }\end{array}$ & $\begin{array}{c}\text { Incidence } \\
\text { density ratio }\end{array}$ & $\begin{array}{c}95 \% \text { confidence } \\
\text { interval }\end{array}$ & $P$-value \\
\hline$\geq 400$ & 4.7 & $(1.6,13.3)$ & 0.004 \\
$200-399$ & 1.4 & $(0.5,4.2)$ & 0.6 \\
$100-199$ & 1.4 & $(0.5,4.0)$ & 0.6 \\
$<100$ & 1.0 & - & - \\
\hline
\end{tabular}


purchasing cattle from outside sources, with the accompanying risk of introducing Salmonella via a subclinical shedder that has been stressed by transport. High cattle density may also be a feature of larger herds and could promote Salmonella transmission; animal crowding not only enhances contact among cattle but may also encourage stressful group dynamics. Alternatively, large herd size may simply equate to a higher number of susceptible animals within the herd. Finally, larger herds may be characterized by management practices that somehow play a role in increasing the incidence of salmonellosis. Herd size is a risk factor that does not easily lend itself to practical intervention because of the management trends and economic constraints that prevail in the modern dairy industry. However, it is possible that certain attributes of larger herds that contribute to their higher Salmonella incidence could in fact be modified to reduce the effect of this disease.

The use of free-stall housing was not a significant predictor of Salmonella incidence in this study, after adjusting for herd size. One study found free-stall housing to be associated with increased odds of fecal Salmonella shedding in a multivariable model that adjusted for herd size (Fossler et al., 2005a), whereas another found a similar association in a univariable analysis but not in a model that controlled for the size of the herd (Huston et al., 2002). Free-stall housing, associated primarily with large herds, presents considerable challenges when combating manure-transmitted pathogens. Freedom of movement in free-stall barns allows cattle to have direct contact with manure from other members of the herd, and it facilitates fecal contamination of common feed and water sources. It is conceivable that this form of housing promotes fecal shedding of Salmonella among dairy cattle in general, including those that are clinically normal, but has a reduced effect on the incidence of salmonellosis.

Neither the use of a commercial gram-negative vaccine nor an owner-reported history of Salmonella during the previous 12 mo was significantly associated with the incidence of salmonellosis in a multivariable Poisson regression model. Other researchers have similarly found that vaccine use (either a gram-negative vaccine or a Salmonella bacterin) and a history of salmonellosis within the past year were not associated with the prevalence of fecal Salmonella shedding when adjusting for herd size in a multivariable model (Huston et al., 2002). A previous history of salmonellosis was unassociated with fecal Salmonella shedding in another study as well (Vanselow et al., 2007). Although we did not find a relationship between immunization practices and the incidence of salmonellosis, it is important to bear in mind that observational studies are not the ideal approach for assessing vaccine efficacy. The lack of an association between salmonellosis and a prior history of Salmonella on the farm was surprising to us, despite the aforementioned prevalence studies. It would seem intuitive for past isolation of the organism to be predictive of future clinical disease, but such was not the case in this study.

\section{CONCLUSIONS}

Although fecal Salmonella shedding has been found to be common at the herd level (Fossler et al., 2004; Blau et al., 2005), the incidence of laboratory-confirmed clinical disease due to Salmonella was comparatively low in this study. Among the positive herds, there was a wide disparity in the incidence of salmonellosis. The animal-level incidence rate was highest for preweaned female calves and lowest for heifers. Salmonella Newport and Typhimurium accounted for about $60 \%$ of the isolates and were widespread among farms; these are also 2 of the most common Salmonella serotypes isolated from people with foodborne infections. Herds with at least 400 female dairy cattle had a higher incidence of salmonellosis than smaller herds, although additional research is needed to clarify the relationship between large herd size and Salmonella.

\section{ACKNOWLEDGMENTS}

The authors thank the veterinarians and dairy herd owners who participated in this study. This project was supported in part by a grant from Pfizer Animal Health.

\section{REFERENCES}

Bender, J. B. 1994. Reducing the risk of Salmonella spread and practical control measures in dairy herds. Bovine Pract. 28:62-65.

Blau, D. M., B. J. McCluskey, S. R. Ladely, D. A. Dargatz, P. J. Fedorka-Cray, K. E. Ferris, and M. L. Headrick. 2005. Salmonella in dairy operations in the United States: Prevalence and antimicrobial drug susceptibility. J. Food Prot. 68:696-702.

CDC (Centers for Disease Control and Prevention). 2007. Preliminary FoodNet data on the incidence of infection with pathogens transmitted commonly through food-10 states, 2006. MMWR Morb. Mortal. Wkly. Rep. 56:336-339.

CDC (Centers for Disease Control and Prevention). 2008a. Outbreak of multidrug-resistant Salmonella enterica serotype Newport infections associated with consumption of unpasteurized Mexicanstyle aged cheese-Illinois, March 2006-April 2007. MMWR Morb. Mortal. Wkly. Rep. 57:432-435.

CDC (Centers for Disease Control and Prevention). 2008b. Preliminary FoodNet data on the incidence of infection with pathogens transmitted commonly through food-10 states, 2007. MMWR Morb. Mortal. Wkly. Rep. 57:366-370.

Cho, S., J. B. Bender, F. Diez-Gonzalez, C. P. Fossler, C. W. Hedberg, J. B. Kaneene, P. L. Ruegg, L. D. Warnick, and S. J. Wells. 2006. Prevalence and characterization of Escherichia coli O157 isolates from Minnesota dairy farms and county fairs. J. Food Prot. 69:252-259.

Cho, S., C. P. Fossler, F. Diez-Gonzalez, S. J. Wells, C. W. Hedberg, J. B. Kaneene, P. L. Ruegg, L. D. Warnick, and J. B. Bender. 2007. 
Antimicrobial susceptibility of shiga toxin-producing Escherichia coli isolated from organic dairy farms, conventional dairy farms, and county fairs in Minnesota. Foodborne Pathog. Dis. 4:178186.

Clegg, F. G., S. N. Chiejina, A. L. Duncan, R. N. Kay, and C. Wray. 1983. Outbreaks of Salmonella Newport infection in dairy herds and their relationship to management and contamination of the environment. Vet. Rec. 112:580-584.

Clinical and Laboratory Standards Institute. 2008. Performance standards for antimicrobial disk and dilution susceptibility tests for bacteria isolated from animals. Approved Standard. 3rd ed. CLSI document M31-A3. Clinical Laboratory Standards Institute, Wayne, PA.

Davison, H. C., A. R. Sayers, R. P. Smith, S. J. Pascoe, R. H. Davies, J. P. Weaver, and S. J. Evans. 2006. Risk factors associated with the Salmonella status of dairy farms in England and Wales. Vet. Rec. 159:871-880.

Divers, T. J., and S. F. Peek. 2008. Rebhun's Diseases of Dairy Cattle. 2nd ed. Saunders Elsevier, St. Louis, MO.

Evans, S., and R. Davies. 1996. Case control study of multipleresistant Salmonella Typhimurium DT104 infection of cattle in Great Britain. Vet. Rec. 139:557-558.

Fossler, C. P., S. J. Wells, J. B. Kaneene, P. L. Ruegg, L. D. Warnick, J. B. Bender, L. E. Eberly, S. M. Godden, and L. W. Halbert. 2005a. Herd-level factors associated with isolation of Salmonella in a multi-state study of conventional and organic dairy farms I. Salmonella shedding in cows. Prev. Vet. Med. 70:257-277.

Fossler, C. P., S. J. Wells, J. B. Kaneene, P. L. Ruegg, L. D. Warnick, J. B. Bender, S. M. Godden, L. W. Halbert, A. M. Campbell, and A. M. Zwald. 2004. Prevalence of Salmonella spp on conventional and organic dairy farms. J. Am. Vet. Med. Assoc. 225:567-573.

Fossler, C. P., S. J. Wells, J. B. Kaneene, P. L. Ruegg, L. D. Warnick, L. E. Eberly, S. M. Godden, L. W. Halbert, A. M. Campbell, C. A. Bolin, and A. M. Zwald. 2005b. Cattle and environmental sample-level factors associated with the presence of Salmonella in a multi-state study of conventional and organic dairy farms. Prev. Vet. Med. 67:39-53

Gay, J. M., and M. E. Hunsaker. 1993. Isolation of multiple Salmonella serovars from a dairy two years after a clinical salmonellosis outbreak. J. Am. Vet. Med. Assoc. 203:1314-1320.

Giles, N., S. A. Hopper, and C. Wray. 1989. Persistence of $S$. Typhimurium in a large dairy herd. Epidemiol. Infect. 103:235241.

Gupta, A., J. Fontana, C. Crowe, B. Bolstorff, A. Stout, S. Van Duyne, M. P. Hoekstra, J. M. Whichard, T. J. Barrett, and F. J. Angulo and The National Antimicrobial Resistance Monitoring System PulseNet Working Group. 2003. Emergence of multidrug-resistant Salmonella enterica serotype Newport infections resistant to expanded-spectrum cephalosporins in the United States. J. Infect. Dis. $188: 1707-1716$

Huston, C. L., T. E. Wittum, B. C. Love, and J. E. Keen. 2002. Prevalence of fecal shedding of Salmonella spp. in dairy herds. J. Am. Vet. Med. Assoc. 220:645-649.

Islam, M., J. Morgan, M. P. Doyle, S. C. Phatak, P. Millner, and X. Jiang. 2004. Persistence of Salmonella enterica serovar Typhimurium on lettuce and parsley and in soils on which they were grown in fields treated with contaminated manure composts or irrigation water. Foodborne Pathog. Dis. 1:27-35.

Kabagambe, E. K., S. J. Wells, L. P. Garber, M. D. Salman, B. Wagner, and P. J. Fedorka-Cray. 2000. Risk factors for fecal shedding of Salmonella in 91 US dairy herds in 1996. Prev. Vet. Med. 43:177-194.
Kwan, P. S., A. Birtles, F. J. Bolton, N. P. French, S. E. Robinson, L. S. Newbold, M. Upton, and A. J. Fox. 2008. Longitudinal study of the molecular epidemiology of Campylobacter jejuni in cattle on dairy farms. Appl. Environ. Microbiol. 74:3626-3633.

Lindberg, A. A., and J. A. Robertsson. 1983. Salmonella Typhimurium infection in calves: Cell-mediated and humoral immune reactions before and after challenge with live virulent bacteria in calves given live or inactivated vaccines. Infect. Immun. 41:751-757.

Mead, P. S., L. Slutsker, V. Dietz, L. F. McCaig, J. S. Bresee, C. Shapiro, P. M. Griffin, and R. V. Tauxe. 1999. Food-related illness and death in the United States. Emerg. Infect. Dis. 5:607-625.

Nielsen, L. R., L. D. Warnick, and M. Greiner. 2007. Risk factors for changing test classification in the Danish surveillance program for Salmonella in dairy herds. J. Dairy Sci. 90:2815-2825.

Peters, A. R. 1985. An estimation of the economic impact of an outbreak of Salmonella Dublin in a calf rearing unit. Vet. Rec. 117:667-668.

Sanchez, S., C. L. Hofacre, M. D. Lee, J. J. Maurer, and M. P. Doyle. 2002. Animal sources of salmonellosis in humans. J. Am. Vet. Med. Assoc. 221:492-497.

Sato, K., P. C. Bartlett, and M. A. Saeed. 2005. Antimicrobial susceptibility of Escherichia coli isolates from dairy farms using organic versus conventional production methods. J. Am. Vet. Med. Assoc. 226:589-594.

Sivapalasingam, S., C. R. Friedman, L. Cohen, and R. V. Tauxe. 2004. Fresh produce: A growing cause of outbreaks of foodborne illness in the United States, 1973 through 1997. J. Food Prot. 67:23422353

Vanselow, B. A., M. A. Hornitzky, K. H. Walker, G. J. Eamens, G. D. Bailey, P. A. Gill, K. Coates, B. Corney, J. P. Cronin, and S. Renilson. 2007. Salmonella and on-farm risk factors in healthy slaughter-age cattle and sheep in eastern Australia. Aust. Vet. J. $85: 498-502$.

Varma, J. K., R. Marcus, S. A. Stenzel, S. S. Hanna, S. Gettner, B. J. Anderson, T. Hayes, B. Shiferaw, T. L. Crume, K. Joyce, K. E. Fullerton, A. C. Voetsch, and F. J. Angulo. 2006. Highly resistant Salmonella Newport-MDRAmpC transmitted through the domestic US food supply: A FoodNet case-control study of sporadic Salmonella Newport infections, 2002-2003. J. Infect. Dis. 194:222-230.

Voetsch, A. C., T. J. Van Gilder, F. J. Angulo, M. M. Farley, S. Shallow, R. Marcus, P. R. Cieslak, V. C. Deneen, and R. V. Tauxe and Emerging Infections Program FoodNet Working Group. 2004 FoodNet estimate of the burden of illness caused by nontyphoidal Salmonella infections in the United States. Clin. Infect. Dis. 38(Suppl. 3):S127-S134.

Warnick, L. D., L. M. Crofton, K. D. Pelzer, and M. J. Hawkins. 2001. Risk factors for clinical salmonellosis in Virginia, USA cattle herds. Prev. Vet. Med. 49:259-275.

Warnick, L. D., K. Kanistanon, P. L. McDonough, and L. Power. 2003. Effect of previous antimicrobial treatment on fecal shedding of Salmonella enterica subsp. enterica serogroup B in New York dairy herds with recent clinical salmonellosis. Prev. Vet. Med. 56:285-297.

Wells, S. J., P. J. Fedorka-Cray, D. A. Dargatz, K. Ferris, and A. Green. 2001. Fecal shedding of Salmonella spp. by dairy cows on farm and at cull cow markets. J. Food Prot. 64:3-11.

Wray, C., and A. Wray. 2000. Salmonella in Domestic Animals. CABI Pub., Wallingford, UK. 